\title{
POWERFUL FLARES FROM RECOILING BLACK HOLES IN QUASARS
}

\author{
G. A. Shields ${ }^{1}$ And E. W. Bonning ${ }^{1,2}$ \\ Received 2007 August 1; accepted 2008 April 11
}

\begin{abstract}
Mergers of spinning black holes can give recoil velocities from gravitational radiation up to several thousand $\mathrm{km} \mathrm{s}^{-1}$. A recoiling supermassive black hole in an AGN retains the inner part of its accretion disk. Marginally bound material rejoining the disk around the moving black hole releases a large amount of energy in shocks in a short time, leading to a flare in thermal soft X-rays with a luminosity approaching the Eddington limit. Reprocessing of the X-rays by the infalling material gives strong optical and ultraviolet emission lines with a distinctive spectrum. Despite the short lifetime of the flare $\left(\sim 10^{4} \mathrm{yr}\right)$, as many as $10^{2}$ flares may be in play at the present time in QSOs at redshifts $\sim 1-3$. These flares provide a means to identify high-velocity recoils.
\end{abstract}

Subject headings: black hole physics - galaxies: active — quasars: general

Online material: color figures

\section{INTRODUCTION}

Simulations of binary black hole mergers show large recoil velocities ("kicks") of the final merged black hole resulting from from anisotropic emission of gravitational radiation (see, e.g., Campanelli et al. [2007] for a summary and references). The highest kick so far computed ${ }^{3}$ is $2500 \mathrm{~km} \mathrm{~s}^{-1}$ for spin $a_{*}=0.8$, with both black hole spins initially perpendicular to the orbital angular momentum and anti-aligned to each other (González et al. 2007; Tichy \& Marronetti 2007). Campanelli et al. (2007) predict a maximum recoil velocity of $4000 \mathrm{~km} \mathrm{~s}^{-1}$ for equal mass black holes with maximal spin in this configuration. For a binary supermassive black hole $\left(\sim 10^{8} M_{\odot}\right)$ formed during a galactic merger (Begelman et al. 1980), the kick may displace the black hole from the galactic nucleus or eject it entirely (Merritt et al. 2004 and references therein). For a recoil occurring in an active galactic nucleus (AGN) with an accretion disk, the inner disk will remain bound to the black hole, providing fuel for continued AGN activity (Loeb 2007; Bonning et al. 2007). Such a "wandering QSO" might be observed as a QSO displaced from the galactic nucleus or as a QSO with emission lines shifted relative to the galactic velocity. However, AGNs rarely show displaced nuclei (Libeskind et al. 2006), and a search for Doppler-shifted broad-line regions by Bonning et al. (2007) failed to detect any strong candidates for recoil. Bogdanović et al. (2007) argue that accretion by the merging black holes will align their spins in a way unfavorable for large kicks.

It is important to explore additional observational signatures of high-velocity recoils that might help to confirm actual cases in nature. We note here that the sudden change in the black hole velocity leads to an increase in the energy of the disk matter that remains bound to the black hole, evaluated in the reference frame of the moving black hole. This can lead to a brief but powerful release of energy in soft X-rays and other forms of radiation (Shields et al. 2007). In $\S 2$ we discuss the mass and energy involved in the reforming disk and the timescale and luminosity of the resulting "recoil flare." In $\S 3$ we present a simple numerical

\footnotetext{
${ }^{1}$ Department of Astronomy, University of Texas, Austin, TX 78712; shields@ astro.as.utexas.edu.

2 YCAA-Department of Physics, Yale University, New Haven, CT 06520; erin.bonning@yale.edu.

${ }^{3}$ Dain et al. (2008) report computed kicks up to $3300 \mathrm{~km} \mathrm{~s}^{-1}$ for $a_{*}=0.925$.
}

simulation of the disk's response to the kick. In $\S 4$ we discuss the observational appearance of the recoil flare, including soft $\mathrm{X}$-ray emission from the primary shocks and secondary emission from photoionized gas and heated dust. In $\S 5$ we estimate the rate of occurrence of these events and the number of recoil flares that may be observable at the present time.

While this manuscript was undergoing revision, a paper appeared by Lippai et al. (2008) also predicting prompt shocks in an accretion disk around a recoiling black hole. Lippai et al. (2008) focus on smaller radii and shorter timescales, suitable for searching for optical counterparts of gravitational wave detections of merging supermassive black holes. Here we discuss the outermost bound disk with longer timescales with an eye to detecting recoil flares currently in play in the AGN population.

\section{ENERGETICS}

The response of the accretion disk to the kick is a strong function of radius. Tightly bound material at radii where the orbital velocity greatly exceeds $v_{\text {kick }}$ will have its orbits modestly perturbed and will shock gently, if at all (Loeb 2007; Lippai et al. 2008). Farther out, marginally bound material will lag substantially behind the recoiling hole and then fall back into the disk on highly eccentric orbits that reach velocity $\sim v_{\text {kick }}$ at periastron. This leads to the expectation of shocks at velocities of order $v_{\text {kick }}$ for the marginally bound material, and a large thermal luminosity because of the large mass and short timescale involved. Because most of the mass of an accretion disk resides at larger radii, a large part of the entire disk may be involved in this phenomenon (see $\S 3$ ).

For a black hole merger taking place in an AGN, the accretion disk fueling the AGN will remain bound to the recoiling black hole inside the radius $R_{b}=\left(10^{18.12} \mathrm{~cm}\right) M_{8} v_{1000}^{-2}$, where the orbital velocity equals $v_{\text {kick}}$. Here $M_{8}=M_{\mathrm{BH}} / 10^{8} M_{\odot}$ and $v_{1000}=$ $v_{\text {kick }} / 1000 \mathrm{~km} \mathrm{~s}^{-1}$. For an $\alpha$ disk (Shakura \& Sunyaev 1973; Frank et al. 2002), the disk mass $M_{b}$ that remains bound is

$$
M_{b}=\left(10^{6.60} M_{\odot}\right) \alpha_{-1}^{-4 / 5} M_{8}^{1 / 4} \dot{M}_{0}^{7 / 10} R_{17}^{5 / 4}
$$

or

$$
M_{b}=\left(10^{8.02} M_{\odot}\right) \alpha_{-1}^{-4 / 5} M_{8}^{3 / 2} \dot{M}_{0}^{7 / 10} v_{1000}^{-5 / 2},
$$


where $\dot{M}_{0}$ is the accretion rate in solar masses per year (Loeb 2007; Bonning et al. 2007). Here $\dot{M}$ refers to the normal AGN phase before the tightening binary opens a gap in the disk at small radii $\sim 10^{2.4} r_{g}$, where $r_{g} \equiv G M_{\mathrm{BH}} / c^{2}$ (Milosavljević \& Phinney 2005; MacFadyen \& Milosavljević 2008; Loeb 2007). The retained disk mass can fuel QSO activity over a disk consumption time $t_{d}$ on the order of the viscous timescale of the outer bound disk where most of the mass resides. Once the inner gap has refilled, the postmerger accretion rate will resemble the premerger rate, giving $t_{d} \approx M_{b} / \dot{M}_{0} \approx\left(10^{8} \mathrm{yr}\right) \alpha_{-1}^{-4 / 5} M_{8}^{3 / 2} \dot{M}_{0}^{-3 / 10} v_{1000}^{-5 / 2}$.

Consider a recoil directed along the rotation axis ( $z$-axis) of the accretion disk. Prior to the recoil, the material at radius $R$ orbits with the Keplerian velocity $v_{\text {Kep }}$, specific angular momentum $l=R v_{\mathrm{Kep}}$, and energy $E=-\frac{1}{2} v_{\mathrm{Kep}}^{2}$. After the kick, the material still has the same angular momentum around the axis of symmetry, but it now has a velocity component $u_{z}=-v_{\text {kick }}$ along the negative $z$-axis in the reference frame moving with the black hole. (We use $\boldsymbol{u}$ for velocities in the rest frame of the postkick black hole.) This velocity adds in quadrature to the orbital velocity $u_{\phi}=v_{\text {Kep }}$. The total energy per unit mass is now $E^{\prime}=$ $E+\frac{1}{2} v_{\text {kick }}^{2}$. This applies at each radius that remains bound to the hole, and therefore the total energy of the disk material has increased by an amount $E_{\text {kick }}=E^{\prime}-E=\frac{1}{2} M_{b} v_{\text {kick}}^{2}$. Because the angular momentum is unchanged, the bound material will eventually settle into circular orbits at the original radius and binding energy. The excess energy that the material has immediately after the kick must therefore be dissipated. We argue below that this dissipation will in large part involve shocks at $\sim v_{\text {kick}}$, leading to an outburst or recoil flare with $E_{\text {flare }}=\xi_{E} E_{\text {kick, }}$, where $\xi_{E}$ is of order unity. The energy associated with a disk with the mass given by equation (2) is

$$
E_{\text {flare }}=\left(10^{57.0} \mathrm{erg}\right) \alpha_{-1}^{-4 / 5} M_{8}^{3 / 2} \dot{M}_{0}^{7 / 10} v_{1000}^{-1 / 2} \xi_{E} .
$$

We have included here the entire bound disk mass. While the mass in the inner disk will acquire excess energy per unit mass of $\frac{1}{2} v_{\text {kick}}^{2}$, this may be dissipated in a less dramatic fashion; but as noted above, much of the bound disk mass will undergo shocks of order $v_{\text {kick}}$.

A more rigorous calculation takes account of the slowing of the black hole as it shares linear momentum with the bound disk mass. When the disk has returned to its equilibrium state, the disk and black hole share a velocity $v_{z}=v_{\text {kick }}\left(1+M_{b} / M_{\mathrm{BH}}\right)^{-1}$, and the energy dissipated is $E_{\text {kick }}=\frac{1}{2} M_{b} v_{\text {kick }}^{2}\left(1+M_{b} / M_{\mathrm{BH}}\right)^{-1}$. The kinetic energy lost by the black hole provides the energy radiated and the kinetic energy associated with the motion of the disk in the $z$-direction. For $M_{b} / M_{\mathrm{BH}}<1$ as considered here, most of the energy goes to radiation. Additional energy will be imparted to disk material outside $R_{b}$ that is drawn toward the moving black hole but remains unbound. We ignore these complications here.

Following a perfectly axial kick, the particles at any radius will follow nonintersecting elliptical orbits in the reference frame of the moving black hole, and a number of orbits may pass before the excess energy is dissipated. For a kick inclined to the disk axis, the available energy will be similar (see $\S 3$ ), but particles initially at radii with $v_{\text {Kep }}$ not greatly larger than $v_{\text {kick }}$ (i.e., $R$ of order $R_{b}$ ) will have a postkick velocity in the black hole's frame that is a strong function of azimuth in the disk. These particles will follow orbits with a range of inclinations and eccentricities, and the disk will be not merely perturbed but seriously disrupted. On approximately the orbital period, material from different parts of the disk will collide and shock at velocities of order $v_{\text {kick}}$. For the mass surface density underlying equation (1), a majority of the bound mass lies outside $R=0.5 R_{b}$, and we expect that much of the excess energy represented by $E_{\text {flare }}$ will be dissipated in shocks at velocities of order $v_{\text {kick }}(\operatorname{see} \S 3)$. The temperature of an AGN disk at relevant radii is $<10^{4} \mathrm{~K}$, with a sound speed $c_{s}<10 \mathrm{~km} \mathrm{~s}^{-1}$. Thus, collisions at even a fraction of $v_{\text {kick }}$ are highly supersonic.

We take the timescale for the recoil flare to be

$$
t_{\text {flare }}=P_{b} \xi_{t}=\left(10^{3.4} \mathrm{yr}\right) M_{8} v_{1000}^{-3} \xi_{t},
$$

where $P_{b}=2 \pi R_{b} / v_{\text {kick }}$ is the Keplerian orbital period at $R_{b}$. The factors $\xi_{E}$ and $\xi_{t}$ express the uncertainty in the radiated energy and timescale. Below we estimate $\xi_{E} \approx 1$ and $\xi_{t} \approx 2$ on the basis of simple numerical simulations. In $\S 4$ we argue that the flare may be observable for $\xi_{E}$ as small as $10^{-2}$.

The power associated with this dissipation is about $E_{\text {flare }} / t_{\text {flare }}$, or

$$
L_{\text {flare }}=\left(10^{46.1} \mathrm{erg} \mathrm{s}^{-1}\right) \alpha_{-1}^{-4 / 5} M_{8}^{1 / 2} \dot{M}_{0}^{7 / 10} v_{1000}^{5 / 2}\left(\xi_{E} / \xi_{t}\right) .
$$

For comparison, the bolometric luminosity of the AGN is

$$
L_{\mathrm{bol}}=\epsilon \dot{M} c^{2} f_{g}=\left(10^{45.8} \mathrm{erg} \mathrm{s}^{-1}\right) \dot{M}_{0} f_{g},
$$

where efficiency $\epsilon=0.1$. The factor $f_{g} \approx 10^{-1}$ allows for suppression of the central accretion rate by the inner gap formed by the binary (MacFadyen \& Milosavljević 2008). The ratio is

$$
L_{\text {flare }} / L_{\text {agn }}=10^{0.3} \xi_{t}^{-1} f_{g}^{-1} \alpha_{-1}^{-4 / 5} M_{8}^{1 / 2} \dot{M}_{0}^{-3 / 10} v_{1000}^{5 / 2} .
$$

Here and below we take $\xi_{E}=1$. The power of the flare can substantially exceed that of the AGN. This is true because a large part of the disk mass shocks on roughly the orbital period, giving a mass shocked per unit time much larger than the central accretion rate, which is driven by the comparatively long viscous timescale. This disparity in timescale offsets the lower energy per unit mass in the shocks, compared with the black hole accretion efficiency of $\sim 0.1 c^{2}$.

The postshock temperature will be

$$
T_{\text {shock }}=\left(10^{6.9} \mathrm{~K}\right)\left(v_{1000}\right)^{2}=(0.7 \mathrm{keV}) v_{1000}^{2}
$$

(Osterbrock \& Ferland 2006). The flare lifetime is shorter for higher recoil velocities, but the luminosity is higher and the $\mathrm{X}$-rays harder.

The disk mass estimated in equation (2) is subject to a number of uncertainties. We have extrapolated the standard $\alpha$-disk surface density $\Sigma$ to radii where the disk is too cool for the assumed opacity to apply. However, $\Sigma$ is a weak function of opacity (Goodman \& Tan 2004). For larger $M_{\mathrm{BH}}$ and lower $v_{\text {kick }}$, the stability limit $M_{b}<M_{\mathrm{BH}}$ becomes important (Loeb 2007). If we let $R_{\lim }$ and $v_{\text {lim }}$ refer to the radius and orbital velocity within which the $\alpha$-disk mass equals $M_{\mathrm{BH}}$, then from equation (1) we have

$$
\begin{aligned}
& R_{\lim }=\left(10^{18.11} \mathrm{~cm}\right) \alpha_{-1}^{16 / 25} M_{8}^{3 / 5} \dot{M}_{0}^{-14 / 25} \\
& v_{\lim }=\left(10^{3.01} \mathrm{~km} \mathrm{~s}^{-1}\right) \alpha_{-1}^{-8 / 25} \mathrm{M}_{8}^{1 / 5} \dot{M}_{0}^{7 / 25}
\end{aligned}
$$

This limits the energy and luminosity of the flare, but it remains a powerful event.

A further complication is the role of the disk's self-gravity at larger radii (Goodman 2003). For an $\alpha$-disk, this comes into play where the Toomre stability parameter reaches unity, $r_{Q} \approx 10^{3.6} r_{g}$, and a Keplerian velocity $v_{Q} \approx 10^{3.7} \mathrm{~km} \mathrm{~s}^{-1}$. The consequences 


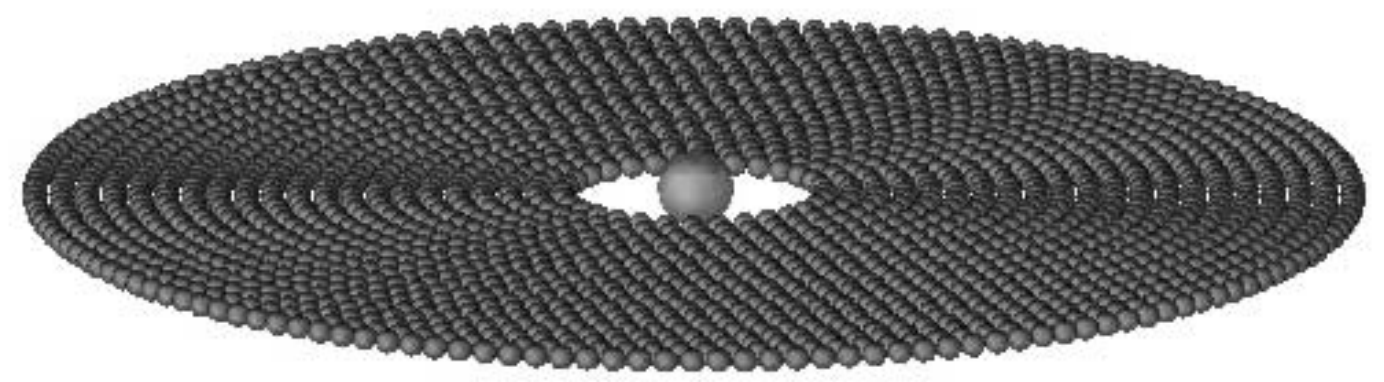

FIG. 1.- Initial configuration $(t=0)$ of simulated accretion disk before recoil of the central black hole (see text). Inner and outer radii are at 10 and 50 in the dimensionless units of the model. Disk rotates counterclockwise seen from above. [See the electronic edition of the Journal for a color version of this figure.]

for the disk structure outside $r_{Q}$ are unclear. If the surface density of the disk is severely reduced at radii $\sim 10^{5} r_{g}$ of interest here, then the amount of shocked gas and the power in the flare will be correspondingly reduced; but clumping of the disk gas should not greatly affect our conclusions. If the disk regulates itself at $Q \approx 1$, then Goodman finds a radial dependence of $\Sigma \propto r^{-1}$ or $r^{-3 / 2}$ rather than the $r^{-3 / 4}$ dependence underlying equation (1). Such a modification of the disk mass near $R_{b}$ would affect our quantitative results but leave the recoil flare a readily observable phenomenon. Goodman summarizes a variety of observational indications that gas disks in AGNs do in fact extend to parsec scales. We proceed on the assumption that a gas disk exists at these radii, but we caution that the mass is uncertain.

\section{NUMERICAL SIMULATION}

In order to test our assumption of high-velocity shocks, we have carried out a simple numerical simulation of the response of the disk to the black hole recoil. We simulated the initial disk as a collection of collisionless particles in Keplerian orbit in the $x-y$ plane and followed the motion of the particles and black hole in three dimensions with the aid of an $N$-body code by Aarseth (1985), adapted to our purposes. We worked in dimensionless units with $G=1$, a particle mass $m_{p}=1$, and $M_{\mathrm{BH}}=10^{5}$. The initial disk had zero thickness. (An AGN disk at the radii of interest has $H / R \ll 1$, and for the nonaxial kicks of interest, postkick motions out of the original plane far exceed the initial disk thickness and internal sound speed.) We ignored the gravitational force of the disk on itself and on the black hole. We modeled the case of a kick in the $y-z$ plane at $45^{\circ}$ to the rotation axis. In the units of our model, the radial limits of the disk were $R_{1}=10$ and $R_{2}=50$. We took $v_{\text {kick }}=65$, giving $R_{b}=23.6$, intermediate between $R_{1}$ and $R_{2}$. Test calculations reproduced a stable disk around a stationary hole, and the nonintersecting nature of orbits for an axial kick. Note that the choice of particle mass and radius scale is arbitrary. The simulation is defined by the direction of the kick and the ratios $R_{1} / R_{b}$ and $R_{2} / R_{b}$.

Scaling of our dimensionless units to specific astrophysical parameters is straightforward using the expressions in $\S 2$. However, because the simulation uses constant $\Sigma$, one must specify a disk mass $M_{d}=\left(10^{7} M_{\odot}\right) M_{d, 7}$. In the physical case, $R_{b}=$ $\left(10^{18.12} \mathrm{~cm}\right) M_{8} v_{1000}^{-2}$, whereas $R_{b}=23.63$ in the simulation. Therefore, radii in the simulation should be multiplied by a factor $\left(10^{16.75} \mathrm{~cm}\right) M_{8} v_{1000}^{-2}$, where $10^{16.75}=10^{18.12} / 23.63$. In like fashion, mass in the model should be multiplied by $\left(10^{3.71} M_{\odot}\right) M_{d, 7}$, velocity by $\left(10^{1.19} \mathrm{~km} \mathrm{~s}^{-1}\right) v_{1000}$, time by $\left(10^{10.56} \mathrm{~s}\right) M_{8} v_{1000}^{-3}$, energy by $\left(10^{49.38} \mathrm{erg}\right) M_{d, 7} v_{1000}^{2}$, power by $\left(10^{38.82} \mathrm{erg} \mathrm{s}^{-1}\right) M_{d, 7} M_{8}^{-1} v_{1000}^{5}$, and angular momentum by $\left(10^{59.94} \mathrm{~g} \mathrm{~cm}^{2} \mathrm{~s}^{-1}\right) M_{d, 7} M_{8} v_{1000}^{-1}$.

In order to test for collisions, we associated a fixed radius $r_{p}$ of unity with each particle. The initial disk comprised concentric rings of particles, separated by $2 r_{p}$ in radius and in azimuth.
Thus, the disk particles are initially just touching their neighbors. This arrangement gave us 1968 particles. We defined a "collision" to occur when two particles passed within $r_{p}$ of each other.

The expected behavior can be visualized in the rest frame of the black hole just after the kick. The particles have velocity components $-v_{\text {kick }, y}$ and $-v_{\text {kick }, z}$ added to their original orbital velocity. The total $y$-component in this frame is $u_{y}=$

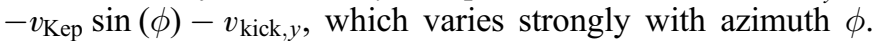
The binding energy per unit mass in the frame of the hole is now $E=-G M_{\mathrm{BH}} / R+\frac{1}{2} u^{2}$, where $u$ is the particle's velocity in the rest frame of the hole. This determines the orbital semimajor axis, $a=2 G M /(-E)$, and period $P=2 \pi a^{3 / 2}(G M)^{-1 / 2}$. For all radii in our simulation, the material in a given ring is bound to the hole for a range of $\phi$ and unbound at other azimuths. Of the 1968 particles in the model disk, 831 remain bound to the hole, so the bound mass is $M_{b 0}=831$. This is more than twice the 381 particles that are initially inside $R_{b}$; for the tilted kick, a substantial part of the mass outside $R_{b}$ remains bound to the black hole, more than offsetting the azimuths at $R<R_{b}$ that are unbound.

In the units of our model, the orbital period at $R_{b}$ is $P_{b}=2.28$, and this sets the timescale of the recoil flare. We ran our simulation from $t=0$ to $t=14$. Figures $1-3$ show the positions of the particles at several times during the evolution. ${ }^{4}$ The more tightly bound material at smaller radii follows the hole relatively closely. More weakly bound material lags before ultimately returning to periastron, where it collides with other portions of the disk material. The bound particles experience collisions at high velocity with other particles originating at substantially different locations in the original disk. We take the energy dissipated per collision to be $\frac{1}{2} v_{\mathrm{rel}}^{2} m_{p}$.

Figure 4 shows the power per unit time produced by the colliding particles, computed by summing the energy for 100 consecutive collisions and dividing by the elapsed time. The power level rises over a time $\sim P_{b}$ and persists for several times $P_{b}$. The power at later times is exaggerated because of repeat collisions involving particles that have completed multiple orbits. The light curve is normalized to $P_{b}$ for the time axis and $L_{0}=\frac{1}{2} M_{b} v_{\text {kick }}^{2} / P_{b}$ for the luminosity axis, where we have used the actual bound mass (831 particles of unit mass) rather than the smaller value of the initial disk mass inside $R_{b}$ (381 particles).

The rms relative velocity of the colliding particles, weighted by the collision energy, rises from $v / v_{\text {kick }}=0.3$ near the start of the run to 0.9 at $t=P_{b}$ and 1.4 for the entire run. This characterizes which shock velocities dominate the power and the shock temperature and spectrum.

Two theoretical light curves are shown for comparison in Figure 4: (1) Here each bound particle was assumed to shock

4 An animation is available at http://www.as.utexas.edu/ shields/recoil/ t65.gif. 


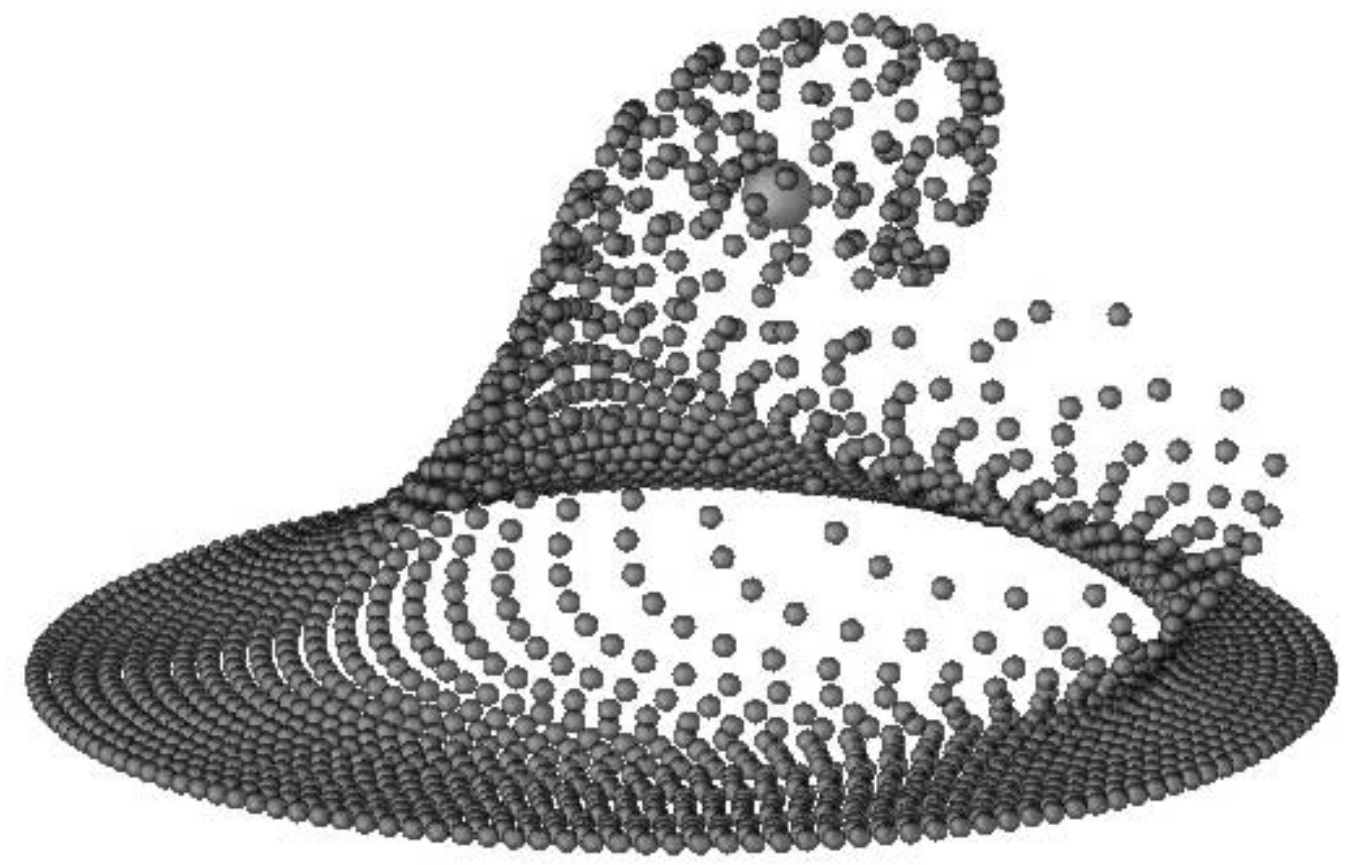

FIg. 2.- Test particle simulation of accretion disk at time $t=1$ after recoil of the central black hole (see text). In the dimensionless units of the model, the recoil has $v_{\text {kick }}=65$ and is inclined $45^{\circ}$ to the initial disk axis. This corresponds to $65 \%$ of the innermost orbital velocity. The prekick Keplerian orbital period is $P_{b}=2.28$ at the radius $R_{b}=23.6$ where $v_{\text {Kep }}=v_{\text {kick }}$. The orbital period at the inner and outer boundaries of the initial disk are $P=0.63$ and 7.0, respectively. [See the electronic edition of the Journal for a color version of this figure.]

exactly one orbital period after the kick, releasing an energy $\frac{1}{2} m_{p} v_{\text {kick. }}^{2}$. The events were ordered in terms of $P$ and binned in groups of 50 particles to calculate the luminosity. This light curve is again normalized by $L_{0}$ computed with the full bound mass in the model. (2) The smooth curve in Figure 4 represents an analytic solution for an axial kick. The postkick orbital period increases monotonically and approaches infinity as $R$ approaches $R_{b}$. We assume that the mass originating at each radius shocks at velocity $v_{\text {kick }}$ at time $t=P$, giving a power $L=\frac{1}{2} v_{\text {kick }}^{2} d M / d t$, where $d M / d t=d M / d P=2 \pi \Sigma R d R / d P$, and $P(R)$ is given by the postkick binding energy in the frame of the moving black hole. This curve is normalized to the mass inside $R_{b}\left(M_{b}=\pi R_{b}^{2} \Sigma\right)$, appropriate for an axial kick in a constant $\Sigma$ disk. For constant surface mass density $\Sigma$, this gives

$$
\dot{M}=\frac{(4 / 3)\left(M_{b} / P_{b}\right) \zeta^{-5 / 3}}{\left(\zeta^{-2 / 3}+1\right)^{3}}
$$

where $\zeta \equiv t / P_{b}$. This decreases as $\dot{M} \propto t^{-5 / 3}$ at late times, a qualitatively similar decline to comparison curve (1) above. This case is given for reference only, as an axial kick will dissipate slowly (see above); and for smaller radii (having $P \ll P_{b}$ ) the orbits are less radically perturbed. Despite the approximations, the various light curves agree at the factor of 2 level as to the power expected at times of 1 to several times $P_{b}$.

Integrated over the run, the power dissipated in collisions is $E_{\text {coll }}=2.8 \times 10^{6}$, somewhat exaggerated by repeat collisions at later times. We can compare this with the overall energetics of the disk. The binding energy of the bound mass immediately after the kick is $E_{b 0}=-1.28 \times 10^{6}$, and its total angular momentum is $L_{b 0}=1.07 \times 10^{6}$. Let us assume conservation of angular momentum for the bound mass in the aggregate as it settles into a circular disk around the recoiling hole. The shallowest binding energy for this mass and angular momentum occurs for a narrow orbiting ring, giving a radius $R_{\text {ring }}=16.6$ and energy $E_{\text {ring }}=$
$-2.51 \times 10^{6}$. In this case, the energy that must be dissipated to reach this configuration is $\Delta E=E_{b 0}-E_{\text {ring }}=1.23 \times 10^{6}$. Alternatively, if the final state is a uniform disk with a sharp outer boundary, then $E_{\text {final }}=(32 / 25) E_{\text {ring }}=-3.21 \times 10^{6}$ and the dissipated energy is $\Delta E=1.93 \times 10^{6}$. These values are comparable to the simple estimate $\Delta E_{0}=\frac{1}{2} M_{b 0} v_{\text {kick }}^{2}=1.76 \times 10^{6}$, using here the actual bound mass for the tilted kick. This supports a value $\xi_{E} \approx 1$ in equation (3).

Light curve " 1 " in Figure 4 by design gives a total energy, integrated over time of $E=\frac{1}{2} M_{b 0} v_{\text {kick. }}^{2}$. The discussion of the preceding paragraph indicates that the actual energy dissipated over the duration of the flare will be close to this amount. The peak luminosity of light curve 1 is $\sim 0.3 L_{0}$, where $L_{0} \equiv \Delta E_{0} / P_{b}$ or $10^{44.71} \mathrm{erg} \mathrm{s}^{-1}$, and this would be reproduced by taking an effective duration of $\sim 3 P_{b}$ for the flare. The light curve based on the particle collision rate in the simulation (Fig. 4, black curve) shows a power level of $\sim 0.13 L_{0}$ at times from $P_{b}$ to $\sim 4 P_{b}$. This lower power level, compared to light curve 1 , may result from collisions that are undercounted by our simple scheme, or from material that takes a number of orbits to suffer a high-velocity collision. In any case, the total energy of the event is determined by the physical considerations above. The greater uncertainty is in the detailed light curve. Note that we have normalized the simulation light curve and light curve 1 to the energy released by the total bound mass in the simulation, which is $2.2=831 / 381$ times larger than the mass $M_{b}$ within $R_{b}$ in the initial disk. The latter, smaller $M_{b}$ is used in $\S \S 2$ and 4 , which are conservative in this sense.

For our model, the most tightly bound quarter of the particles have a postkick orbital period $P<1.1 P_{b}$, one-half of all the bound particles have period $P<2.4 P_{b}$, and the most weakly bound quarter of the particles have $P>5.4 P_{b}$. This suggests that, to characterize the main surge of the flare, we can take $\xi_{t} \approx 2$ in equation (4). There is some material with arbitrarily weak binding energy after the kick. This material lags far behind 


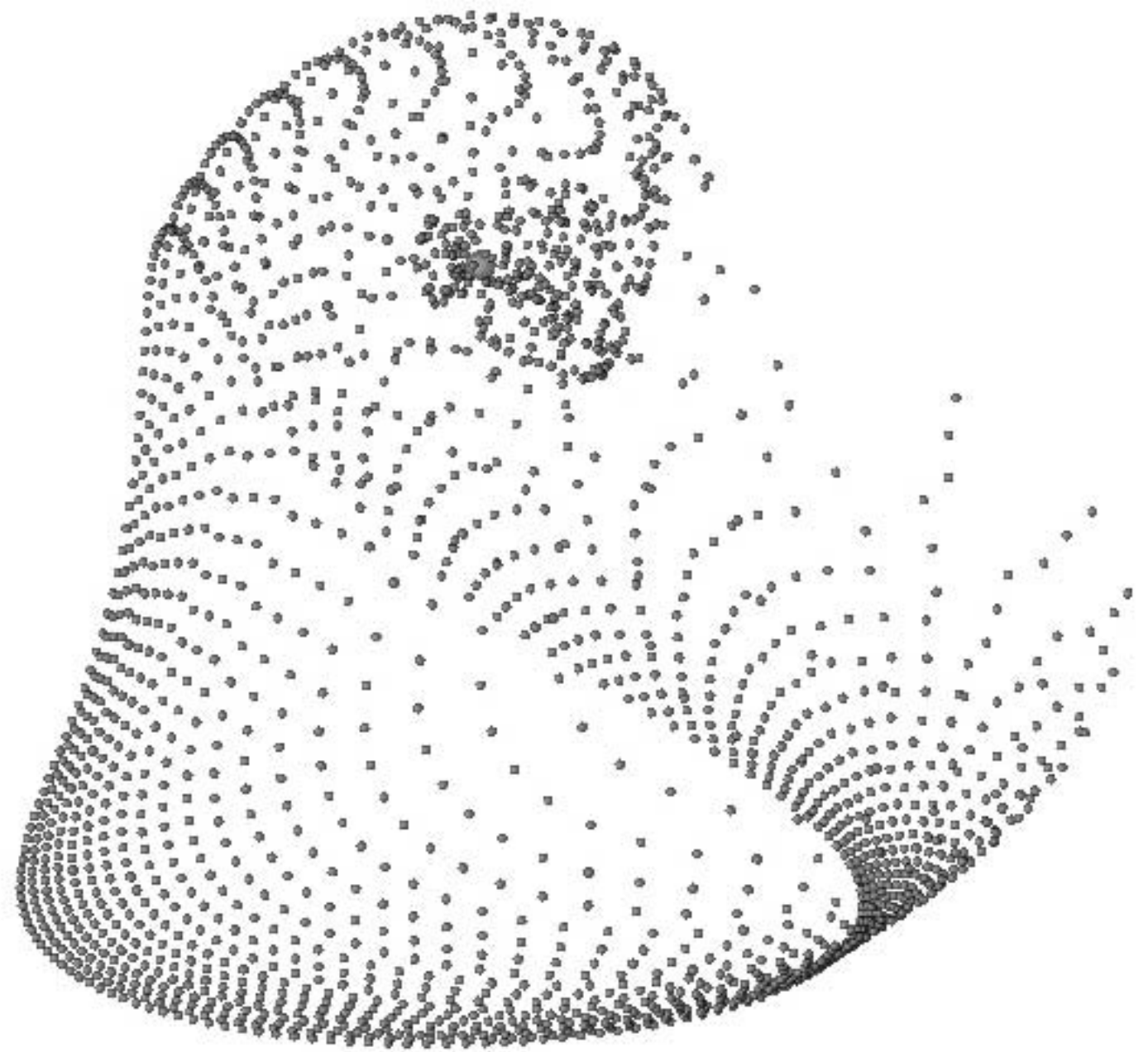

Fig. 3. - Simulated accretion disk at time $t=2.5=1.1 P_{b}$ after recoil of central black hole (see text). [See the electronic edition of the Journal for a color version of this figure.]

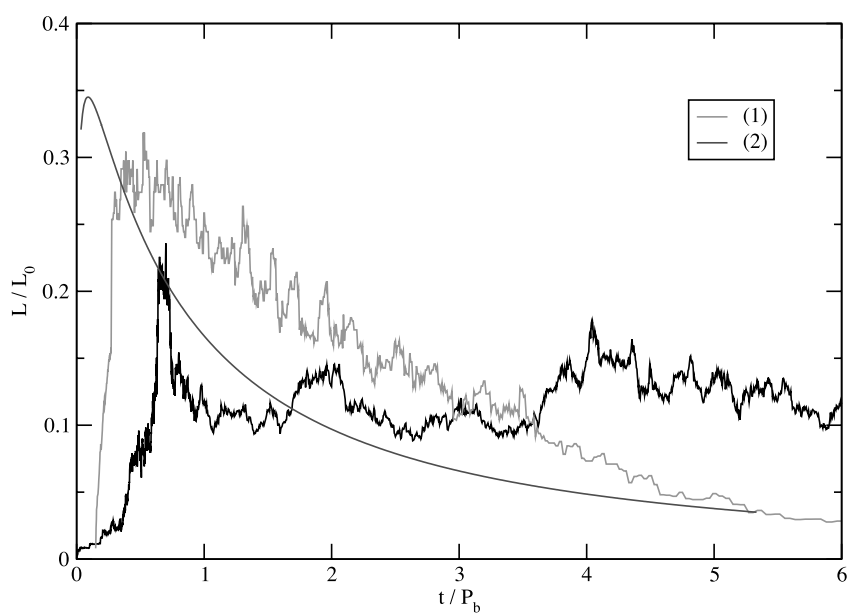

Fig. 4.-Predicted light curves for recoil flare (see text). Black curve with sustained luminosity is power per unit time for particle collisions in numerical simulation; power at later times is overestimated because of repeat collisions. Green curve 1 with declining power is based on postkick orbital period $P$ of each bound particle, assuming that an energy $\frac{1}{2} m_{p} v_{\text {kick }}^{2}$ is dissipated at $t=P$. Smooth red curve 2 is analytic solution for axial kick. Each curve is normalized to $P_{b}$ for the time axis and $L_{0}=\frac{1}{2} M_{b} v_{\text {kick }}^{2} / P_{b}$ for the luminosity axis. For reference, $P_{b}=$ $10^{3.4} \mathrm{yr}$ and $L_{0}=10^{45.7} \mathrm{erg} \mathrm{s}^{-1}$ for $M_{\mathrm{BH}}=10^{8} M_{\odot}, \dot{M}=10^{-0.5} M_{\odot} \mathrm{yr}^{-1}$, and $v_{\text {kick }}=1000 \mathrm{~km} \mathrm{~s}^{-1}$ (eqs. [4] and [5]). [See the electronic edition of the Journal for a color version of this figure.] the moving hole, giving a prolonged tail to the impact rate. In our numerical model, the bulk of the shock power corresponds to collisions occurring at radii near or inside $R_{b}$; the mean collision radius, weighted by collision energy, is $\sim 12$ or $0.5 R_{b}$, with little variation during the run. The collisions typically involve pairs of bound particles that originate at substantially different radii near or outside $R_{b}$, and substantially different azimuths. These particles move on quite eccentric orbits, and the collisions producing most of the power typically occur not far from periastron.

We conclude that a nonaxial kick will severely disrupt the orbits of the mass elements in the disk at radii $\sim R_{b}$, leading to strong shocks. A physically realistic hydrodynamical simulation will be needed to compute a more detailed light curve. However, our simulation supports the approximate energetics outlined in $\S 2$.

\section{APPEARANCE}

Equation (8) suggests that shocked gas in the postkick disk will release its energy largely in the form of thermal soft X-rays, with $k T \approx 0.7 \mathrm{keV}$ for $v_{\text {kick }}=1000 \mathrm{~km} \mathrm{~s}^{-1}$, or $0.2 \mathrm{keV}$ for $v_{\text {kick }}=500 \mathrm{~km} \mathrm{~s}^{-1}$. Subject to the redshift and absorption by the surrounding material, this radiation could be observable.

Consider a QSO with $M_{\mathrm{BH}}=10^{8} M_{\odot}$ accreting at $10^{-0.5} M_{\odot} \mathrm{yr}^{-1}$ (giving $L / L_{\mathrm{Ed}} \approx 10^{-0.8}$ ) before the inner gap forms. Then $v_{\text {lim }}=750 \mathrm{~km} \mathrm{~s}^{-1}$, and for a kick velocity of $1000 \mathrm{~km} \mathrm{~s}^{-1}$ the bound disk mass of $10^{7.7} M_{\odot}$ is less than $M_{\mathrm{BH}}$. The radius of the retained disk in this case is $R_{b}=10^{18.1} \mathrm{~cm}$, and 


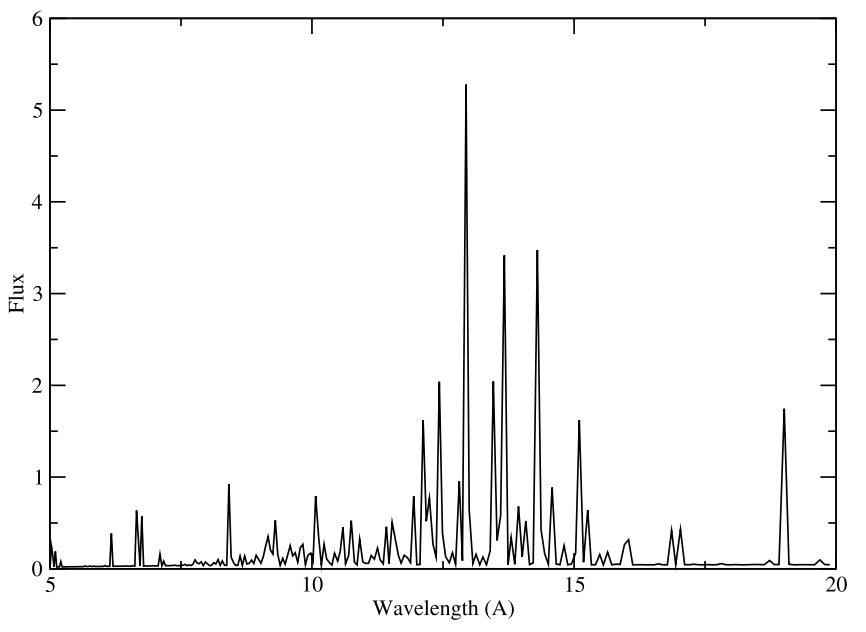

FIG. 5.- Spectrum of optically thin gas in coronal equilibrium at $T=10^{7} \mathrm{~K}$ in wavelength range 5-20 $\AA$ (see text). Note strong emission lines of Fe xx and neighboring ions. Abscissa is wavelength in $\AA$ in bins $1500 \mathrm{~km} \mathrm{~s}^{-1}$ wide; ordinate is $\lambda F_{\lambda}$ in arbitrary units. The intrinsic line width for the plot is set at $1000 \mathrm{~km} \mathrm{~s}^{-1}$.

the surface density is $\Sigma \approx M_{b} / \pi R_{b}^{2}=10^{4.2} \mathrm{~g} \mathrm{~cm}^{-2}$. The above discussion suggests that, for times $t>P_{b}$, the recoil flare has the character of material on highly eccentric orbits impacting the disk at radii $\sim R_{b}$. For the parameters just assumed, $t_{\text {flare }} \approx \xi_{t} P_{b}=$ $\left(10^{3.4} \mathrm{yr}\right) \xi_{t}$ or $\sim 5000 \mathrm{yr}$ for $\xi_{t}=2$. The corresponding mass infall rate is $\dot{M}_{\text {flare }} \approx M_{b} / t_{\text {flare }}=\left(10^{4.0} M_{\odot} \mathrm{yr}^{-1}\right)$. This large rate of mass undergoing shocks is, of course, the basis of the large power of the flare. The density of the infalling material is subject to uncertain geometrical details, but a simple estimate is $\rho \approx$ $\dot{M}_{\text {flare }} /\left(2 \pi R_{b}^{2} v\right)=10^{-15.2} \mathrm{~g} \mathrm{~cm}^{-3}$, or $N=\rho / m_{\mathrm{H}}=\left(10^{8.6} \mathrm{~cm}^{-3}\right)$. The cooling time will be $\sim 10^{-2} \mathrm{yr}$, so that the material will cool in a relatively narrow zone on the scale of $R_{b}$. The local energy flux in the X-ray flare is $F_{\text {flare }} \approx \frac{1}{2} \rho v^{3} \approx 10^{8.5} \mathrm{erg} \mathrm{cm}^{-2} \mathrm{~s}^{-1}$. The column density in the impacting stream is $\sim \rho R_{b} \approx 10^{2.9} \mathrm{~g} \mathrm{~cm}^{-2}$. The optical depth due to photoelectric absorption by $\mathrm{H}, \mathrm{He}$, and heavy elements will be large, and the X-rays may be unable to penetrate the enveloping inflow. However, the luminosity of the shocked flow, $L_{\text {flare }} \approx\left(10^{45.4} \mathrm{erg} \mathrm{s}^{-1}\right)$, is close to the Eddington limit. This raises the possibility of radiation-driven outflows, although the energy available is insufficient to expel the entire disk mass. Given these outflows and the complex geometry of the infalling material, there may be clear lines of sight on which the X-rays escape. While the shock temperature is high, the effective temperature corresponding to $F_{\text {flare }}$ is only $T_{\text {eff }}=$ $\left(F_{\text {flare }} / \sigma\right)^{1 / 4} \approx 10^{3.2} \mathrm{~K}$. This underscores the sensitivity of the observed appearance of the flare to the nature of the reprocessing that occurs in the surrounding material.

For an estimate of the spectrum of the cooling shocked gas, we computed a coronal equilibrium model using version 07.02.00 of the photoionization code CLOUDY, most recently described by Ferland et al. (1998). We used $T=10^{7} \mathrm{~K}, N=10^{9.6} \mathrm{~cm}^{-3}$, and solar abundances. (This corresponds to a preshock velocity of $1100 \mathrm{~km} \mathrm{~s}^{-1}$ and density of $10^{9} \mathrm{~cm}^{-3}$.) Much of the total cooling is thermal bremsstrahlung. There are many strong emission lines, including lines of Fe XVII to Fe XX at 12-15 $\AA$, each with several percent of the total energy (see Fig. 5). If not totally absorbed by the infalling gas, this should be recognizable as a unique spectrum for a point source in a galactic nucleus. Comptonization should be insignificant, and the flare will not emit hard X-rays. There could, however, be hard X-rays if the AGN luminosity is not entirely shut off by the inner gap in the disk at the time of the merger.
TABLE 1

Line Intensities of Shock Precursor

\begin{tabular}{|c|c|c|c|}
\hline Ion & $\begin{array}{c}\lambda \\
(\AA)\end{array}$ & $\mathrm{T} 7.0$ & T6.3 \\
\hline $\mathrm{H} \beta$ & 4861 & 1.000 & 1.000 \\
\hline $\mathrm{H} \alpha \ldots \ldots \ldots \ldots \ldots$ & 6563 & 5.0 & 6.5 \\
\hline 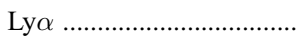 & 1215 & 28.7 & 88.5 \\
\hline Не І..................................... & 5876 & 0.10 & 0.24 \\
\hline 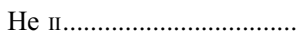 & 304 & 17.1 & 19.9 \\
\hline 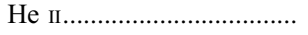 & 1640 & 2.7 & 5.4 \\
\hline Не пा & 4686 & 0.31 & 0.68 \\
\hline C & 2326 & 1.83 & 0.69 \\
\hline C III & 1909 & 5.1 & 14.9 \\
\hline C III & 977 & 2.8 & 2.9 \\
\hline C IV & 1549 & 25.3 & 55.3 \\
\hline N IV (.................................... & 1486 & 1.58 & 5.1 \\
\hline $\mathrm{N}$ v & 1240 & 3.52 & 1.65 \\
\hline 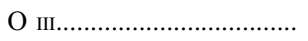 & 1665 & 2.0 & 4.7 \\
\hline O IV ................................... & 1402 & 2.1 & 4.8 \\
\hline $\mathrm{O} \vee$ & 1218 & 8.6 & 1.6 \\
\hline $\mathrm{O}$ V & 630 & 5.1 & 0.03 \\
\hline O VI & 1035 & 48.0 & 0.44 \\
\hline Mg ІІ & 2798 & 5.2 & 5.9 \\
\hline $\mathrm{Si}$ III & 1397 & 1.6 & 3.6 \\
\hline $\log N_{\mathrm{H}} \ldots \ldots \ldots \ldots$ & & 9.0 & 8.0 \\
\hline 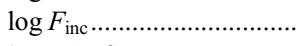 & & 8.77 & 6.70 \\
\hline $\log F(\mathrm{H} \beta) \ldots \ldots \ldots$ & & 6.34 & 4.21 \\
\hline 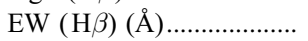 & & 780 & 660 \\
\hline 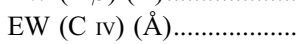 & & 6100 & 11,500 \\
\hline
\end{tabular}

Note-Emission-line intensities relative to $\mathrm{H} \beta$ of CLOUDY models for shock precursor zone photoionized by coronal emission spectrum at $10^{7.0}$ or $10^{6.3} \mathrm{~K}$ (see text). Incident and $\mathrm{H} \beta$ flux in erg $\mathrm{cm}^{-2} \mathrm{~s}^{-1}$. Equivalent widths relative to nebular continuum only.

The X-ray luminosity will photoionize the infalling material approaching the shock. The local energy flux in the X-ray flare directed into the infalling gas is $F_{\text {flare }} \approx 10^{8.8} \mathrm{erg} \mathrm{cm}^{-2} \mathrm{~s}^{-1}$, giving an ionizing photon flux of $\phi_{i} \approx 10^{18.2} \mathrm{~cm}^{-2} \mathrm{~s}^{-1}$ and an ionization parameter $U=\phi_{i} / N c=0.05$. We have computed photoionization models with CLOUDY for this precursor zone. We used the coronal model emission as the ionizing spectrum with flux $10^{8.8} \mathrm{erg} \mathrm{cm}^{-2} \mathrm{~s}^{-1}$, a gas density $N=10^{9} \mathrm{~cm}^{-3}$, and a cutoff column density $10^{23.5} \mathrm{~cm}^{-2}$. The photoionized depth in this preshock flow is $\sim 10^{13.7} \mathrm{~cm}$, again narrow compared to $R_{b}$. The high-ionization parameter and hard ionizing spectrum give strong ultraviolet emission lines of highly ionized species (see Table 1 and Figs. 6 and 7). The Ly $\alpha$ line carries $11 \%$ of the incident energy flux, and $\mathrm{O}$ VI and $\mathrm{C}$ IV are comparably strong. The $\mathrm{H} \beta$ and $\mathrm{C}$ Iv lines, respectively, have equivalent widths of $\sim 780$ and $6100 \AA$ in terms of the diffuse continuum of the precursor alone. The Balmer and helium lines are affected by large optical depths. Based on the coronal equilibrium model, the shock will contribute an additional $12 \%$ to the continuum at $\mathrm{H} \beta$ and $30 \%$ at $\lambda 1550$, slightly reducing the equivalent widths. The high density suppresses forbidden line emission. Aside from this and transfer effects in some lines, the spectrum is not strongly sensitive to density, because the shock velocity alone determines the post-shock temperature and also the ionization parameter of the precursor.

We also computed CLOUDY models for a case corresponding to $v_{\text {kick }}=500 \mathrm{~km} \mathrm{~s}^{-1}$. We took $M_{8}=10^{-0.5}$ and $\dot{M}_{0}=10^{-0.7}$ to conform to the self-gravity limit. This gives $t_{\text {flare }}=10^{4.1} \mathrm{yr}$ for $\xi_{t}=2$, and a preshock gas density of $N=10^{8.1} \mathrm{~cm}^{-3}$ estimated as above. Table 1 shows the emission-line intensities of the 


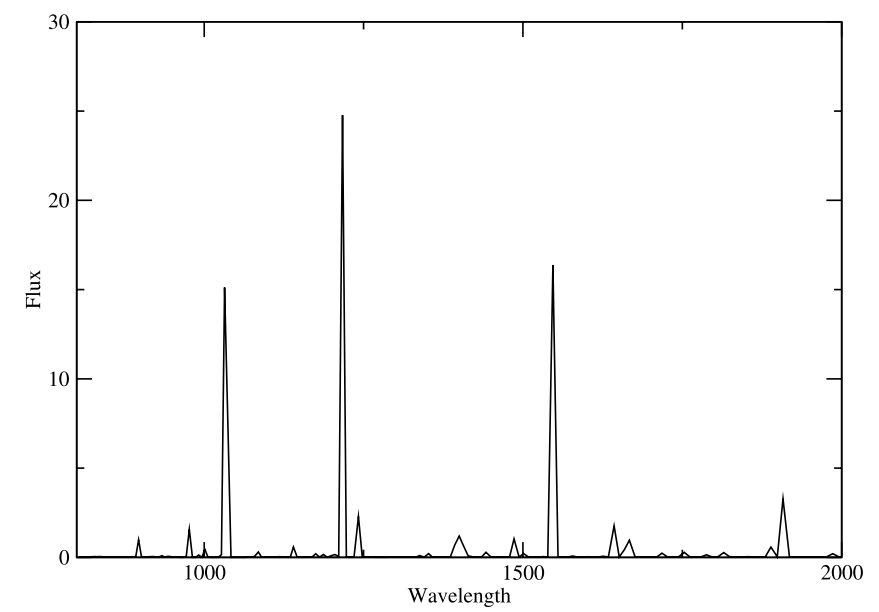

FIG. 6.- Spectrum of photoionized precursor for $T_{\text {shock }}=10^{7} \mathrm{~K}$ in wavelength range 800-2000 $\AA$ (see text). Note strong emission lines of o VI, Ly $\alpha$, and $\mathrm{C}$ Iv. Flux is $\lambda F_{\lambda}$ in arbitrary units.

precursor ionized by the emission from a $10^{6.3} \mathrm{~K}$ coronal model for the shocked gas. Many of the same strong emission lines occur, such as $\mathrm{H} \beta$, $\mathrm{Ly} \alpha$, and $\mathrm{C}$ IV. However, $\mathrm{O}$ vI is much weaker, due to a lower level of ionization and a lower electron temperature. Thus, the high-ionization line intensities offer a potential diagnostic of the shock velocity and hence $v_{\text {kick}}$. Forbidden lines such as [O III] 25007 , [O III] $\lambda 4363$, and [ $\mathrm{Ne} \mathrm{v}] \lambda 3426$ have intensities of $0.5-1.0$ times $I(\mathrm{H} \beta)$ because of the lower density.

We can estimate the relative luminosity of the recoil flare at specific wavelengths with the aid of equation (7). In the optical, a typical AGN energy distribution gives $\lambda L_{\lambda}(5100)=0.11 L_{\text {bol }}$ (Kaspi et al. 2000). The photoionized precursor by itself has $\lambda L_{\lambda}(5100)=0.03 L_{\text {bol }}^{\text {flare }}$ based on the CLOUDY model. Using these factors in equation (7), we have

$$
\lambda L_{\lambda}^{\text {flare }} / \lambda L_{\lambda}^{\mathrm{AGN}}=C_{\lambda} \xi_{t}^{-1} f_{g}^{-1} \alpha_{-1}^{-4 / 5} M_{8}^{1 / 2} \dot{M}_{0}^{-3 / 10} v_{1000}^{5 / 2},
$$

where $C_{\lambda}=10^{-0.3}$ for $\lambda 5100$. A similar calculation for $\lambda 1550$ gives $C_{\lambda}=10^{-0.6}$. Therefore, the flare continuum is comparable with the AGN continuum if $f_{g}=1$ or dominant if $f_{g}$ is small. This in turn implies that the equivalent widths of the flare emission lines remain large relative to the total observed continuum, including the AGN component. In the composite QSO spectrum of Vanden Berk et al. (2001) the equivalent widths of $\mathrm{H} \beta, \mathrm{C}$ IV, Ly $\alpha$, and $\mathrm{O}$ vi are 46, 24, 93, and $10 \AA$, respectively, mostly in the broad-line component. With their large equivalent widths and narrow profiles, the flare emission lines should be prominent in the total spectrum. Indeed, they remain conspicuous even if the flare contribution is 2 orders of magnitude weaker than estimated here, relative to the AGN. The number of detectable flares in play could be increased by as much as an order of magnitude by the prolonged arrival of weakly bound material catching up to the black hole (see $\S 3$ ). The line widths will be $\sim v_{\text {kick }}$, typically $500-1000 \mathrm{~km} \mathrm{~s}^{-1}$ for flares likely to be observed (see below). These line widths resemble those of narrow-line Seyfert 1 (NLS1) objects or the lines from the traditional narrow-line region (NLR) of AGNs. However, the lines of the recoil flare will be distinctive, showing (1) potentially large equivalent widths, (2) weakness or absence of forbidden lines such as [O III] 25007 , (3) strong He II $\lambda 4686$ and possibly $\mathrm{O}$ vi $\lambda 1035$, and (4) likely velocity shifts and asymmetries of order $v_{\text {kick }}$. Absorption by dust in the surrounding disk material may be important. The disk temperature

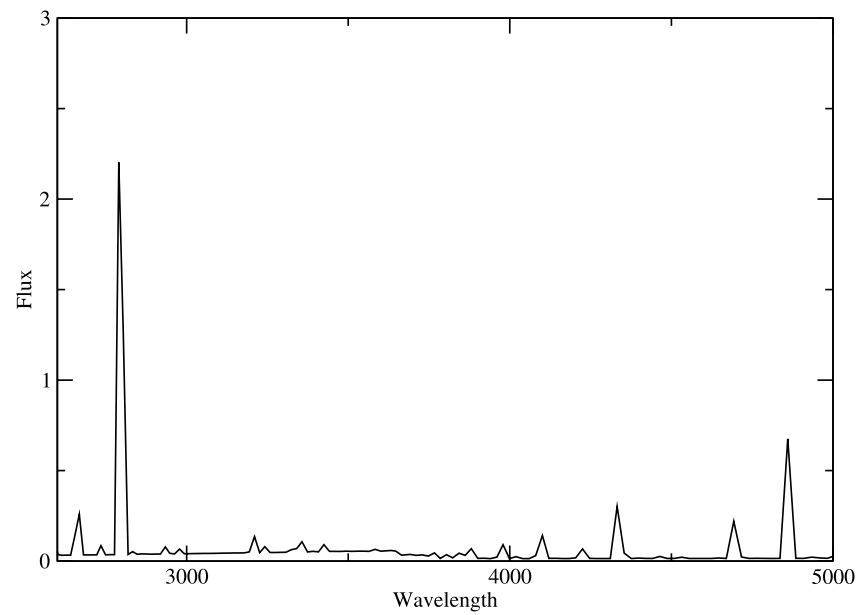

FIG. 7.- Spectrum of photoionized shock precursor for $T_{\text {shock }}=10^{7} \mathrm{~K}$ in wavelength range $2600-5000 \AA$ (see text). Flux is $\lambda F_{\lambda}$ in arbitrary units. Note lines of $\mathrm{H} \beta$, He II $\lambda 4686$, and $\mathrm{Mg}$ II.

near $R_{b}$ is low enough before the kick for dust to exist, and the energy flux from the shocks may not be enough to evaporate refractory grains.

We can similarly estimate the prominence of the soft X-rays from the primary shocked gas. For the AGN continuum at $0.3 \mathrm{keV}$, Laor et al. (1997) find an average $\nu L_{\nu, 0.3}=10^{-1.0} \nu L_{\nu}(3000 \AA)$, and by the above conversions, this gives $\nu L_{\nu, 0.3}=10^{-1.8} L_{\mathrm{bol}}^{\mathrm{AGN}}$. From the CLOUDY model for $T=10^{7} \mathrm{~K}$, we find $R_{0.3} \equiv$ $\nu L_{\nu, 0.3}^{\text {flare }} / L_{\text {bol }}^{\text {flare }}=10^{-1.0}$. Combining these expressions with equation ( 7$)$, we find

$\nu L_{\nu, 0.3}^{\text {flare }} / \nu L_{\nu, 0.3}^{\mathrm{AGN}}=10^{1.1}\left(R_{0.3} / 10^{-1}\right) \xi_{t}^{-1} f_{g}^{-1} \alpha_{-1}^{-4 / 5} M_{8}^{1 / 2} \dot{M}_{0}^{-3 / 10} v_{1000}^{5 / 2}$.

If a substantial fraction of the X-ray radiation from the shocks is able to reach the observer, the object will stand out as an exceptionally strong source in soft X-rays, relative to its optical and ultraviolet luminosity. This remains true for $v_{\text {kick }}=500 \mathrm{~km} \mathrm{~s}^{-1}$, giving $T_{\text {shock }}=10^{6.3} \mathrm{~K}$. Our coronal equilibrium model at this temperature shows a continuum dropping at $\lambda<30 \AA$ and many emission lines forming a pseudocontinuum at $30-60 \AA$. In the vicinity of $40 \AA(0.3 \mathrm{keV})$, the power in the lines corresponds to $R_{0.3}=10^{-0.5}$, again giving a large ratio of soft X-rays to optical continuum.

The unified model of AGNs posits a thick dusty torus that surrounds the emitting core at a radius of 1 or several parsecs (Antonucci \& Miller 1985). This torus will intercept and reprocess a substantial fraction of the X-ray and optical-ultraviolet radiation from the reforming accretion disk. A large part of the flare luminosity should therefore appear as infrared radiation from dust.

\section{RATE OF OCCURRENCE}

The number of recoil flares currently observable depends on the rate of mergers, the probability of kicks of various velocities, and the flare duration at a given velocity. The kick velocity depends on the magnitude and orientation of the spin of the two black holes and the ratio of their masses. Large kicks occur for rapidly spinning holes with favorable orientations and nearly equal masses. Black holes in AGNs are believed to spin rapidly, with spin parameter $a_{*} \equiv J / M>0.8$, as discussed by Fabian 
(2005), Wang (2006), and references therein. Spin orientations are uncertain and are typically assumed to be random, although they can be affected by accretion (Bogdanović et al. 2007). The distribution of mass ratios is also uncertain. In merger history simulations, Sesana et al. (2007) find that mass ratios in mergers are uniformly distributed in the range $q \equiv m_{2} / m_{1}=0.1-1$ for low-mass seed black holes, whereas large seeds favor more equal mergers.

Let $d N / d t$ be the merger rate per year of observer time in some range of $M_{\mathrm{BH}}$ and redshift $z$, and let $f_{v}$ be the fraction of mergers giving a kick velocity greater than $v$. The number of kicks currently observable is roughly $N_{\text {flare }} \approx(d N / d t)\left[t_{\text {flare }}(1+z)\right] f_{v}$, where the factor $1+z$ allows for the observed duration of a flare with intrinsic duration $t_{\text {flare }}$. For mergers of two black holes with equal spin parameter $a_{*}=0.9$ with random orientations, and mass ratios distributed in the range $q=0.2$ to 1 , Schnittman \& Buonanno (2007) find $f_{500}=0.31$ and $f_{1000}=0.08$. For similar assumptions, Baker et al. (2008) find a value $f_{1000}=0.23$. These results rely on uncertain assumptions about the merger details but provide an approximate basis for estimating the number of highvelocity recoils. Below we conservatively use $f_{1000}=0.08$.

Two alternative estimates of $N_{\text {flare }}$ are as follows:

1. Sesana et al. $(2004,2007)$ and Volonteri $(2007)$ compute binary merger rates in hierarchical merger simulations normalized to the observed QSO luminosity function. M. Volonteri (2007, private communication) has kindly provided merger rates for mass ratios $q>0.2$ for the various seed black hole scenarios of Sesana et al. (2007). In the "VHM" scenario (small seeds), the event rate per observer year for $1<z<3$ is $\sim 0.08 \mathrm{yr}^{-1}$ for $\left(m_{1}+m_{2}\right)$ in the range $10^{7}-10^{8} M_{\odot}$ and $0.014 \mathrm{yr}^{-1}$ in the range $10^{8}-10^{9} M_{\odot}$. The event rate is about 3 times smaller in the other scenarios. The rates drop rapidly for $z<1$. Let $f_{\mathrm{Qso}}$ be the fraction of these mergers that are sufficiently gas-rich to fuel QSO activity. For the $10^{7}-10^{8} M_{\odot}$ range, we have $t_{\text {flare }} \approx 800 \xi_{t} \mathrm{yr}$ at $v_{\text {kick }}=1000 \mathrm{~km} \mathrm{~s}^{-1}$ for $M_{\mathrm{BH}}=10^{7.5} M_{\odot}$. Then in the VHM scenario we expect to see roughly $15 \xi_{t} f_{\mathrm{QSO}}$ events currently in play for $f_{1000}=0.08$. For $v_{\text {kick }}>500 \mathrm{~km} \mathrm{~s}^{-1}$, we expect $\sim 500 \xi_{t} f_{\mathrm{QSO}}$ events in play, taking into account the longer flare duration and the larger fraction of recoils above the lower kick velocity. For $10^{8}-10^{9} M_{\odot}$, the limit $M_{b}<M_{\mathrm{BH}}$ comes into play for a typical value $L / L_{\mathrm{Ed}}=10^{-0.5}$ (e.g., Salviander et al. 2007), and this affects $t_{\text {flare }}$. If we take $t_{\text {flare }} \approx 2 \pi R_{\text {lim }} / v_{\text {lim }}$ (eqs. [9] and [10]), then we expect $\sim 8 f_{\text {QSO }}$ flares in play above $1000 \mathrm{~km} \mathrm{~s}^{-1}$ and $\sim 33 f_{\text {QSO }}$ above $500 \mathrm{~km} \mathrm{~s}^{-1}$. These numbers could be several times larger if the disk extends beyond $R_{\text {lim }}$ with a mass remaining below $M_{\mathrm{BH}}$.

2. Haehnelt (1994) derived the black hole merger rate from the QSO luminosity function assuming $L=L_{\mathrm{Ed}}$, one event per black hole, and a QSO lifetime of $t_{\mathrm{QSO}}=10^{7.6} \mathrm{yr}$ (the "Salpeter time" for black hole growth). At $M_{\mathrm{BH}}$ between $10^{7}$ and $10^{8} M_{\odot}$, Haehnelt finds 0.016 events per year of observer time for $z<3$, giving roughly $3 \xi_{t}$ flares in play for $v_{\text {kick }}>1000 \mathrm{~km} \mathrm{~s}^{-1}$, and $94 \xi_{t}$ in play above $500 \mathrm{~km} \mathrm{~s}^{-1}$. Uncertainties in this estimate of $N_{\text {flare }}$ include the assumed value of $L / L_{\mathrm{Ed}}$ and the QSO lifetime, the flare duration, and the number of obscured QSOs. However, we do not need the factor $f_{\mathrm{QSO}}$ because the calculation is based on observed QSOs.

Despite the uncertainties, it appears likely that several flares above $10^{7} M_{\odot}$ are currently observable. We emphasize that this rests on the assumption that the black hole merger occurs while the QSO accretion disk is in place.

For a $10^{8} M_{\odot}$ hole in a QSO shining at $0.3 L_{\mathrm{Ed}}$ at $z=2$ (prior to formation of the inner gap), the received flux from the flare is $\sim 10^{-12.8} \mathrm{erg} \mathrm{cm}^{-2} \mathrm{~s}^{-1}$ for $\xi_{t}=2$, subject to the escape fraction. The observed spectrum will be softened by the redshift, but a considerable fraction of the escaping radiation should still be received at photon energies above $0.2 \mathrm{keV}$. At $10^{-12.8} \mathrm{erg} \mathrm{cm}^{-2} \mathrm{~s}^{-1}$, Hasinger et al. (2005) find $\sim 2$ soft X-ray sources $(0.5-2 \mathrm{keV})$ per square degree, so that the recoil flares would need to be identified from among roughly $10^{5}$ other sources of similar flux. The unique spectrum of the flare should aid recognition.

An optical search for recoil flares could target the strong, narrow emission lines in the rest optical and ultraviolet. For $t_{\mathrm{QSO}}=$ $10^{7.6} \mathrm{yr}$, the fraction of QSOs in the recoil flare stage from equation (4) is

$$
N_{\text {flare }} / N_{\text {QSO }}=10^{-3.2} M_{8} v_{1000}^{-3} \xi_{t} f_{v} .
$$

This gives $N_{\text {flare }} / N_{\mathrm{QSO}}=10^{-4.3} M_{8} \xi_{t}$ for $v_{1000}=1$ and $10^{-3.7} M_{8} \xi_{t}$ for $v_{1000}=0.5$. Thus, recoil flares may be observable in about one in 10,000 QSOs, and one or more examples may be present in existing large spectroscopic surveys. For example, the SDSS DR5 QSO Catalog (Schneider et al. 2007) contains 77,429 objects with a median redshift $z=1.48$ over $\sim 5740 \mathrm{deg}^{2}$ of sky. About $10^{4}$ of these objects have $z<0.8$ so that $\mathrm{H} \beta$ is accessible, and many have $z>1.7$ so that $\mathrm{C}$ IV is accessible. The higher redshift objects typically have relatively high luminosity and black hole masses $\sim 10^{8.5} M_{\odot}$ or higher, so that the self-gravity limit on the accretion disk is significant. However, the discussion in $\S 4$ suggests that, even if the disk mass around $R_{b}$ is much less than that assumed in equation (5), the flare emission lines could still be prominent. The observed QSO population serves as the parent population for the recoil flares, but the AGN luminosity may be dimmed several magnitudes by the inner gap at the time of the recoil. This will complicate the detection of AGN undergoing recoil flares and their inclusion in QSO surveys.

\section{CONCLUSION}

Merged black holes with high recoil velocities can carry a large mass of gas in an accretion disk that remains bound to the black hole. The kick velocity gives the disk material a boost in energy, relative to the moving hole, that will in substantial part be dissipated in shocks within a few orbital periods as the more weakly bound material rejoins the moving disk. This results in a soft X-ray flare lasting thousands of years with a luminosity rivaling or exceeding the AGN luminosity. The X-rays will be heavily absorbed, at least along some lines of sight, and the energy re-emitted in line and continuum emission from photoionized gas and infrared radiation from heated dust. A number of recoil flares may be currently observable. Observational detection of such an event would provide an important confirmation of the occurrence in nature of high-velocity recoils. Detailed simulations of the observational appearance of these recoil flares are needed to aid in identifying such objects in surveys at X-ray and other wavelengths.

We thank Marta Volonteri for providing numerical results and Ski Antonucci, Omer Blaes, Richard Matzner, Milos Milosavljević, and Meg Urry for helpful discussions. E. W. B. acklowledges support by Marie Curie Incoming European Fellowship contract MIF1-CT-2005-008762 within the 6th European Community Framework Programme. 
Aarseth, S. J. 1985, in Multiple Timescales, ed. J. U. Brackbill \& B. I. Cohen (Orlando: Academic Press), 377

Antonucci, R. R. J., \& Miller, J. S. 1985, ApJ, 297, 621

Baker, J. G., Boggs, W. D., Centrella, J., Kelly, B. J., McWilliams, S. T., Miller, M. C., \& van Meter, J. R. 2008, preprint (arXiv:0802.0416)

Begelman, M. C., Blandford, R. D., \& Rees, M. J. 1980, Nature, 287, 307

Bogdanović, T., Reynolds, C. S., \& Miller, M. C. 2007, ApJ, 661, L147

Bonning, E. W., Shields, G. A., \& Salviander, S. 2007, ApJ, 666, L13

Campanelli, M., Lousto, C., Zlochower, Y., \& Merritt, D. 2007, Phys. Rev. Lett., 98, 231102

Dain, S., Lousto, C. O., \& Zlochower, Y., 2008, preprint (arXiv:0803.0351)

Fabian, A. C. 2005, Ap\&SS, 300, 97

Ferland, G. J., Korista, K. T., Verner, D. A., Ferguson, J. W., Kingdon, J. B., \& Verner, E. M. 1998, PASP, 110, 761

Frank, J., King, A., \& Raine, D. J. 2002, Accretion Power in Astrophysics (3rd ed.; Cambridge: Cambridge Univ. Press)

González, J. A., Hannam, M., Sperhake, U., Brügmann, B., \& Husa, S. 2007, Phys. Rev. Lett., 98, 231101

Goodman, J. 2003, MNRAS, 339, 937

Goodman, J., \& Tan, J. C. 2004, ApJ, 608, 108

Haehnelt, M. G. 1994, MNRAS, 269, 199

Hasinger, G., Miyaji, T., \& Schmidt, M. 2005, A\&A, 441, 417

Kaspi, S., et al. 2000, ApJ, 533, 631

Laor, A., Fiore, F., Elvis, M., Wilkes, B., \& McDowell, J. C. 1997, ApJ, 477, 93
EFERENCES

Libeskind, N. I., Cole, S., Frenk, C. S., \& Helly, J. C. 2006, MNRAS, 368, 1381

Lippai, A., Frei, Z., \& Haiman, Z. 2008, preprint (arXiv:0801:0739)

Loeb, A. 2007, Phys. Rev. Lett., 99, 041103

MacFadyen, A. I., \& Milosavljevic, M. 2008, ApJ, 672, 83

Merritt, D., Milosavljević, M., Favata, M., Hughes, S. A., \& Holz, D. E. 2004, ApJ, 607, L9

Milosavljević, M., \& Phinney, E. S. 2005, ApJ, 622, L93

Osterbrock, D. E., \& Ferland, G. J. 2006, Astrophysics of Gaseous Nebulae and Active Galactic Nuclei (2nd ed.; New York: University Science Books)

Salviander, S., Shields, G. A., Gebhardt, K., \& Bonning, E. W. 2007, ApJ, 662, 131

Schneider, D. P., et al. 2007, AJ, 134, 102

Schnittman, J. D., \& Buonanno, A. 2007, ApJ, 662, L63

Sesana, A., Haardt, F., Madau, P., \& Volonteri, M. 2004, ApJ, 611, 623

Sesana, A., Volonteri, M., \& Haardt, F. 2007, MNRAS, 377, 1711

Shakura, N. I., \& Sunyaev, R. A. 1973, A\&A, 24, 337

Shields, G. A., Bonning, E. W., \& Salviander, S. 2007, preprint (arXiv:0707:3625)

Tichy, W., \& Marronetti, P. 2007, Phys. Rev. D, 76, 061502

Vanden Berk, D., et al. 2001, AJ, 122, 549

Volonteri, M. 2007, ApJ, 663, L5

Wang, J.-M. 2006, ApJ, 642, L111 